\title{
Fickian crossover and length scales from two point functions in supercooled liquids
}

Cite as: J. Chem. Phys. 125, 064505 (2006); https://doi.org/10.1063/1.2221309

Submitted: 23 September 2005 . Accepted: 15 June 2006. Published Online: 08 August 2006

Daniel A. Stariolo, and Gabriel Fabricius

\section{ARTICLES YOU MAY BE INTERESTED IN}

Decoupling of exchange and persistence times in atomistic models of glass formers The Journal of Chemical Physics 127, 211101 (2007); https://doi.org/10.1063/1.2803062

Spontaneous and induced dynamic fluctuations in glass formers. I. General results and dependence on ensemble and dynamics

The Journal of Chemical Physics 126, 184503 (2007); https://doi.org/10.1063/1.2721554

Distribution of diffusion constants and Stokes-Einstein violation in supercooled liquids

The Journal of Chemical Physics 140, 224505 (2014); https://doi.org/10.1063/1.4882066

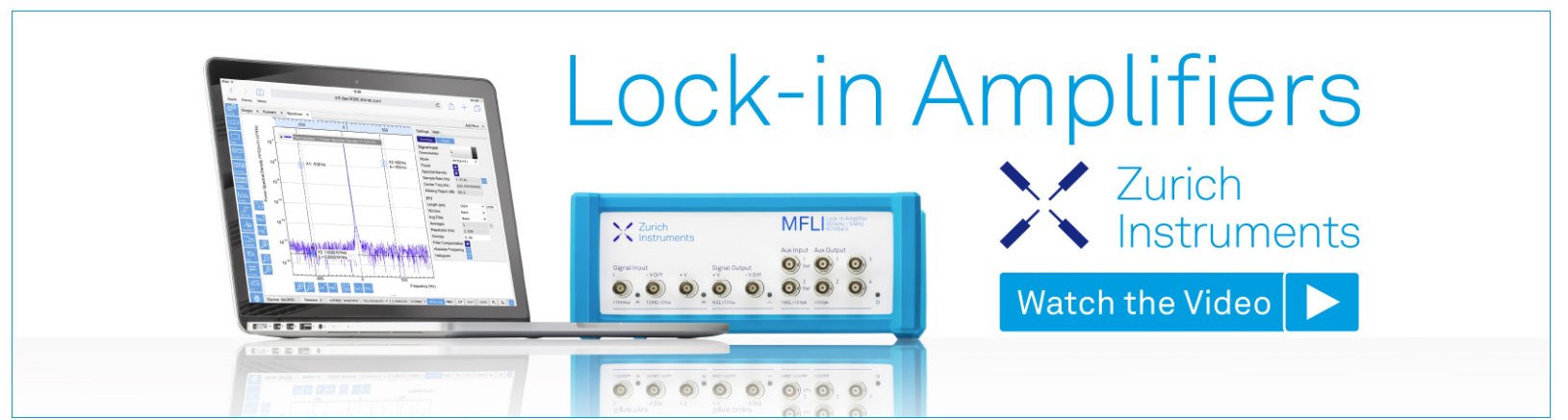

J. Chem. Phys. 125, 064505 (2006); https://doi.org/10.1063/1.2221309 


\title{
Fickian crossover and length scales from two point functions in supercooled liquids
}

\author{
Daniel A. Stariolo ${ }^{\text {a) }}$ \\ Departamento de Física, Universidade Federal do Rio Grande do Sul, CP 15051, \\ 91501-970 Porto Alegre, Brazil \\ Gabriel Fabricius ${ }^{\text {b) }}$ \\ Departamento de Física, Universidad Nacional de La Plata, cc 67, 1900 La Plata, Argentina
}

(Received 23 September 2005; accepted 15 June 2006; published online 8 August 2006)

\begin{abstract}
Particle motion of a Lennard-Jones supercooled liquid near the glass transition is studied by molecular dynamics simulations. We analyze the wave vector dependence of relaxation times in the incoherent self-scattering function and show that at least three different regimes can be identified and its scaling properties determined. The transition from one regime to another happens at characteristic length scales. The length scale associated with the onset of Fickian diffusion corresponds to the maximum size of heterogeneities in the system, and the characteristic time scale is several times larger than the alpha relaxation time. A second crossover length scale is observed, which corresponds to the typical time and length of heterogeneities, in agreement with results from four point functions. The different regimes can be traced back to the behavior of the van Hove distribution of displacements, which shows a characteristic exponential regime in the heterogeneous region before the crossover to Gaussian diffusion and should be observable in experiments. Our results show that it is possible to obtain characteristic length scales of heterogeneities through the computation of two point functions at different times. () 2006 American Institute of Physics.

[DOI: $10.1063 / 1.2221309]$
\end{abstract}

\section{INTRODUCTION}

The basic mechanisms of diffusion in supercooled liquids and glasses are still poorly understood. Despite a considerable amount of work in the last years, different approaches to the problem do not always agree and raise doubts as to the ultimate relevance of the approaches themselves. In the landscape approach ${ }^{1-9}$ the basic hypothesis is that, at low temperatures, the dynamics of a supercooled liquid or a glass is ruled by the complex topography of the potential energy surface, in particular by the multiplicity and complexity of local minima and saddles. The glass transition in this context can be viewed as the manifestation of a particular topological transition in the landscape, at which the mechanism of diffusion by escape through unstable directions ceases to be efficient and hopping through barriers becomes the dominant mechanism to get out of a restricted region in phase space. While this approach has been considerably successful and offered deep insights into the nature of the dynamics in the supercooled regime, its connection with real space particle dynamics is not direct, and limited information on the elementary dynamical processes at the particle level has been obtained. At a more coarse grained level, minimal models with kinetic constraints have been very successful in order to get intuition and insight into elementary dynamical processes. ${ }^{10-15}$ In this kind of model the real space

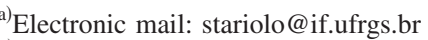

${ }^{b)}$ Research Associate of the Abdus Salam International Center for Theoretical Physics, Strada Costiera 11, Trieste, Italy. Electronic mail: fabriciu@ fisica:unlp.edu.ap
}

dynamics can be analyzed in great detail and relevant mechanisms of the microscopic dynamics can be uncovered. There is no underlying landscape and this has raised the question of the relevance and necessity of a landscape based description of the dynamics of supercooled liquids. Nevertheless, in spite of their success in identifying many qualitative features of supercooled liquids, the extreme simplification in their own definition poses a limit in the description of many complex phenomena, like aging or thermodynamic properties of glasses.

Mode coupling theory $\mathrm{MCT}^{16-18}$ is perhaps the most successful theoretical approach to the dynamics of the supercooled liquid state. It predicts the existence of relevant regimes for relaxation, like the beta and alpha relaxations, and makes quantitative predictions for temperatures above the glass transition. Because of the complex interplay between space and time scales in a supercooled liquid, most studies based on MCT have focused on time scales at fixed length scales, e.g., the analysis of the alpha relaxation time scale is usually done focusing on the wave vector corresponding to the peak of the structure factor. Even the emergence of stretched exponential relaxation, a benchmark of glassy dynamics, is obtained from MCT only through fitting of numerical solutions of the complex set of equations describing time correlations. The origin of stretched exponential relaxation in supercooled liquids is still debated. A common scenario opposes a possibly homogeneously stretched relaxation, which basically means that local regions in supercooled liquids relax in the same time scale, with an heterogeneous scenario in which different regions relax with 
different characteristic times, probably exponentially, giving rise to a global stretching of the relaxation. Only recently the local dynamics has begun to be accessible experimentally, through the emergence of new sophisticated experimental techniques ${ }^{19-21}$ and the concept of dynamical heterogeneities has gained force. ${ }^{19,22}$ It has been realized that complex spatiotemporal correlations characterize an heterogeneous system, which can be described by four point functions, correlations between two points in space at two different times. $^{23-26}$ These dynamical susceptibilities show a peak at a time scale which corresponds to the typical heterogeneity in the system and this time scale grows when temperature is lowered toward the glass transition. An associated growing length scale suggests a situation similar to a critical phenomenon. This length scale is difficult to measure. Experimental results point to small or moderate values of the characteristic length of heterogeneities near the glass transition ${ }^{19,22}$ and computer simulation results are inconclusive due to the limitations in the times scales reached by numerical experiments, when compared to real experiments. ${ }^{25,27}$

A clear signature of dynamical heterogeneity is the observation of two sets of particles with different degree of mobility, which allows one to define "slow particles" and "fast particles" over particular time intervals. ${ }^{20,21,28-30}$ These two sets show up, for example, in a double peak structure of the distributions of particle displacements, and several indicators have been defined in order to locate the time at which this separation is maximal. This time scale is another way of defining the typical lifetime of heterogeneities. At very long time scales the supercooled liquid recovers the characteristics of homogeneous Brownian motion and the distribution of displacements becomes Gaussian. Consequently indicators of non-Gaussian behavior serve to characterize dynamical heterogeneity. ${ }^{30,31}$

Recently, ${ }^{15,32}$ performing a detailed study of the interplay between time and length scales, it was realized that it is possible to obtain characteristic length scales of the process of diffusion in supercooled liquids by analyzing two point functions, namely the self part of the van Hove distribution function $G_{s}(\boldsymbol{r}, t)$ and its Fourier transform, the self incoherent scattering function $F_{s}(\boldsymbol{k}, t)$, the two most common functions used to study dynamical behavior in liquids. ${ }^{33}$ Analyzing the behavior of kinetically constrained lattice models, it was realized that it is possible to define a length scale corresponding to the onset of Fickian diffusion in a supercooled liquid. Above this length the system behaves as a simple fluid and below it persistence dominates and nearly frozen regions are observed up to times corresponding to the alpha relaxation time, precluding the glass transition. This interesting observation, that stretching is dominated by persistence events up to a time in which Fickian diffusion sets in, can only be a rough approximation to the true behavior of a strongly correlated supercooled liquid, as we will see in the following.

In this paper we show results of molecular dynamics simulations of a Lennard-Jones binary mixture (LJBM), focusing on the behavior of the van Hove and self scattering functions through a wide spectrum of time and length scales. ${ }^{34,35}$ Our main result is that characteristic length scales can be unequivocally obtained through the analysis of the wave vector dependence of relaxation times in two point time dependent correlation functions. We have been able to characterize at least three regimes: ballistic, heterogeneous, and Fickian. In particular, the scale characteristic of the onset of Fickian diffusion is shown to correspond to the maximum size of heterogeneities. The other length scale corresponds to the typical heterogeneous behavior and the corresponding time is the typical relaxation time scale. The heterogeneous regime is characterized by stretched behavior of the scattering function and we have observed a corresponding exponential regime in the van Hove distribution function of displacements.

Our results show that typical length scales of heterogeneities can be obtained through analysis of two point functions, like the incoherent scattering function, which are easily obtained experimentally.

In Sec. II we introduce the model, the simulation details, and show the appearance of the different regimes in the inherent structures version of the van Hove distribution function. In Sec. III we present our main results on the wave vector dependence of relaxation times and determination of characteristic time and length scales from analysis of the self incoherent scattering function. In Sec. IV we make a comparison with known experimental results. In Sec. V we present some conclusions.

\section{THE LENNARD-JONES BINARY MIXTURE AND BASIC OBSERVABLES}

We performed molecular dynamics simulations on a well-known LJBM with $80 \%$ particles of type $A$ and $20 \%$ particles of type $B$ with $\epsilon_{A A}=1.0, \epsilon_{B B}=0.5, \epsilon_{A B}=1.5, \sigma_{A A}$ $=1.0, \sigma_{B B}=0.88, \sigma_{A B}=0.8$ at a density 1.204. Most results correspond to systems with $N=1000$ particles. Some results with 130 and 10000 particles will be shown for discussing fine size effects. We used a cutoff radius $R_{c}=1.8$ for the potential and obtained $T_{\mathrm{MCT}} \approx 0.46$ from extrapolation of diffusivity data. The simulations shown here were done at a single working temperature $T=0.525=1.14 T_{\mathrm{MCT}}$, at which the system was equilibrated. As our main aim is to analyze the diffusion dynamics of particles we map the instantaneous configurations to the nearby local minima, called inherent structures. ${ }^{2,36}$ Periodically along a trajectory we take a configuration and let it relax with a conjugate gradient algorithm to the nearest local minimum. In this way we get a map of the true trajectory to a trajectory between local minima. As the temperature is lowered toward the glass transition, trapping in the basins of these inherent structures becomes important and rule the dynamics. ${ }^{4,8,37}$ Nevertheless throughout the exposition we make comparisons with the corresponding results from the real (instantaneous) molecular dynamics trajectories.

In Fig. 1 we compare the behavior of the self incoherent scattering function:

$$
F_{s}(\boldsymbol{k}, t)=\frac{1}{N} \sum_{i=1}^{N} \exp \left[i \boldsymbol{k} \cdot\left(\boldsymbol{r}_{i}(t)-\boldsymbol{r}_{i}(0)\right)\right]
$$

calculated from the real instantaneous configurations, with the same function calculated from the corresponding inherent 


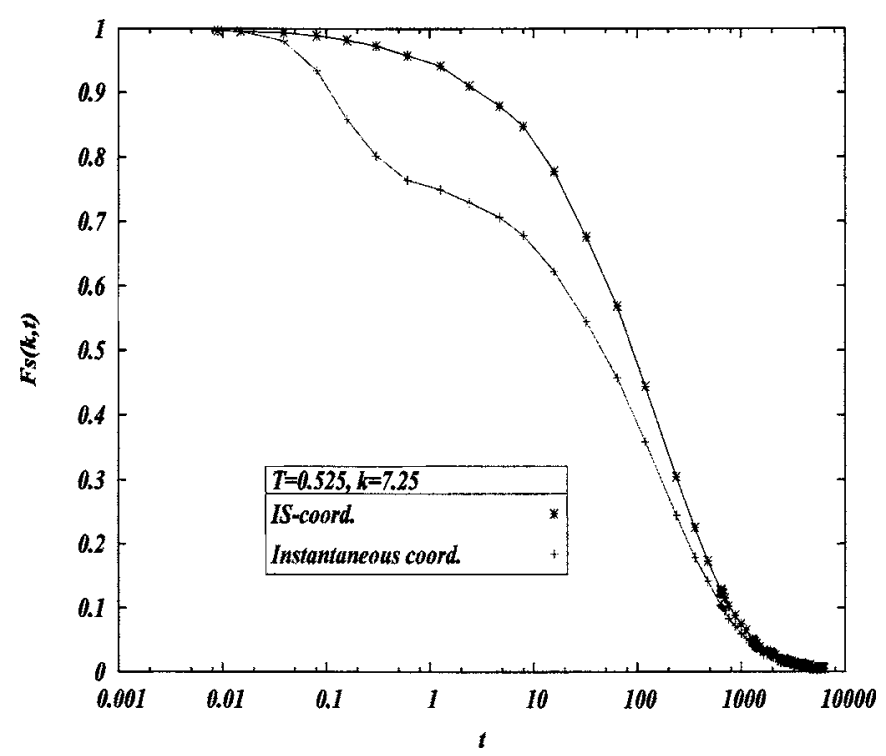

FIG. 1. Self incoherent scattering function for the LJBM at $T=0.525$ for a wave vector corresponding to the peak of the structure factor $k=7.25$, from instantaneous and inherent structures coordinates.

structures. From its definition this function is wave vector dependent. In this figure the wave vector corresponds to the maximum of the structure factor $|k|=7.25$. The main difference between the two curves is the suppression of the relaxation to the plateau in the IS dynamics. This basically means that vibrations of the particles and rattling inside cages is strongly suppressed in the IS dynamics and we are left only with structural displacements. This is good for our purposes of looking at diffusion dynamics at low temperatures. From the instantaneous curve we extract as usual the $\alpha$-relaxation time scale which is approximately $\tau \simeq 120$ for our system.

The same behavior is observed in the mean squared displacement (MSD) of the particles $R^{2}(t)=(1 / N) \sum_{i=1}^{N} \mid \boldsymbol{r}_{i}(t)$ $-\left.\boldsymbol{r}_{i}(0)\right|^{2}$ shown in Fig. 2. Note that the MSD from IS proceeds without the arrest at intermediate times. The two

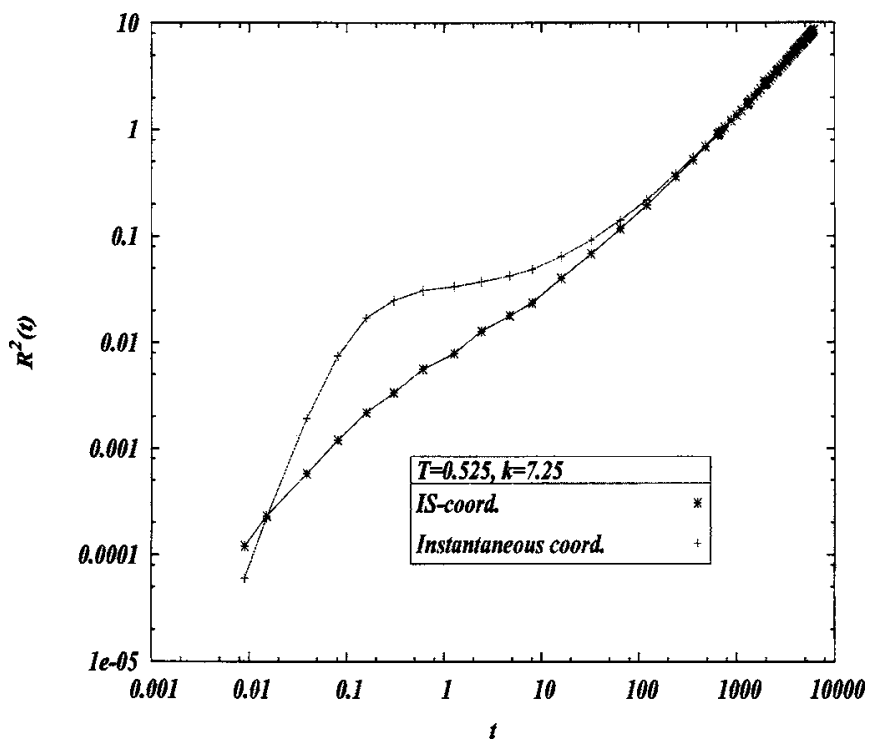

FIG. 2. Mean squared displacement at $T=0.525$ for instantaneous and inherent structure coordinates.

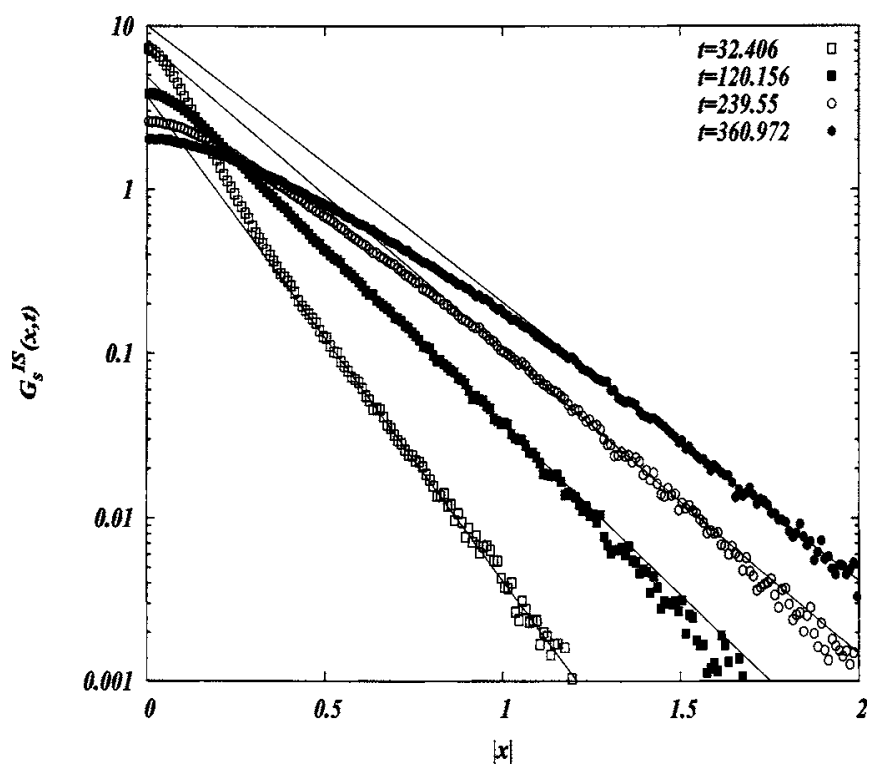

FIG. 3. The van Hove distribution in the exponential regime. Continuous lines are exponential fits.

curves merge approximately around the $\alpha$-relaxation time, from where the system gradually enters a normal diffusive dynamics.

The basic quantity to characterize the displacements of particles with time is the van Hove distribution function. ${ }^{33,34}$ This function has been extensively analyzed in the context of dynamical heterogeneities in supercooled liquids and colloidal systems. ${ }^{20,21,28-30}$ We consider the self part of the function and sum the contributions to the displacements in the three coordinate directions obtaining an effective onedimensional quantity defined by

$$
\begin{aligned}
G_{s}^{\mathrm{IS}}(x, t) & =\frac{1}{N}\left\langle\sum_{i=1}^{N} \delta\left(x-\left|x_{i}^{\mathrm{IS}}(t)-x_{i}^{\mathrm{IS}}(0)\right|\right)\right\rangle \\
& \rightarrow \frac{1}{(4 \pi D t)^{1 / 2}} \exp \left(-\frac{x^{2}}{4 D t}\right),
\end{aligned}
$$

where the superscript IS means that we are considering inherent structure coordinates. The long time, large displacement limit corresponds to homogeneous Fickian diffusion. We were able to distinguish at least two regimes. In Fig. 3 it is shown that an exponential decay fits correctly the data for intermediate time scales and distances $0.5 \leqslant x \leqslant 2$. We will see later when analyzing the wave vector dependence of relaxation times that this regime of times and distances corresponds to the heterogeneous or stretched dynamical regime. An exponential decay in the distribution of displacements was also observed by Vogel et al. ${ }^{38}$ and Schroeder et al. ${ }^{39}$ when analyzing the displacements of particles between two consecutive inherent structures. In Fig. 4 we show the asymptotic Gaussian regime of the van Hove function. Note that it is necessary to wait for times several orders longer than the alpha scale in order that almost all particles enter a Fickian regime. This fact was recently observed and analyzed in Ref. 31. The crossover length and time scales be- 


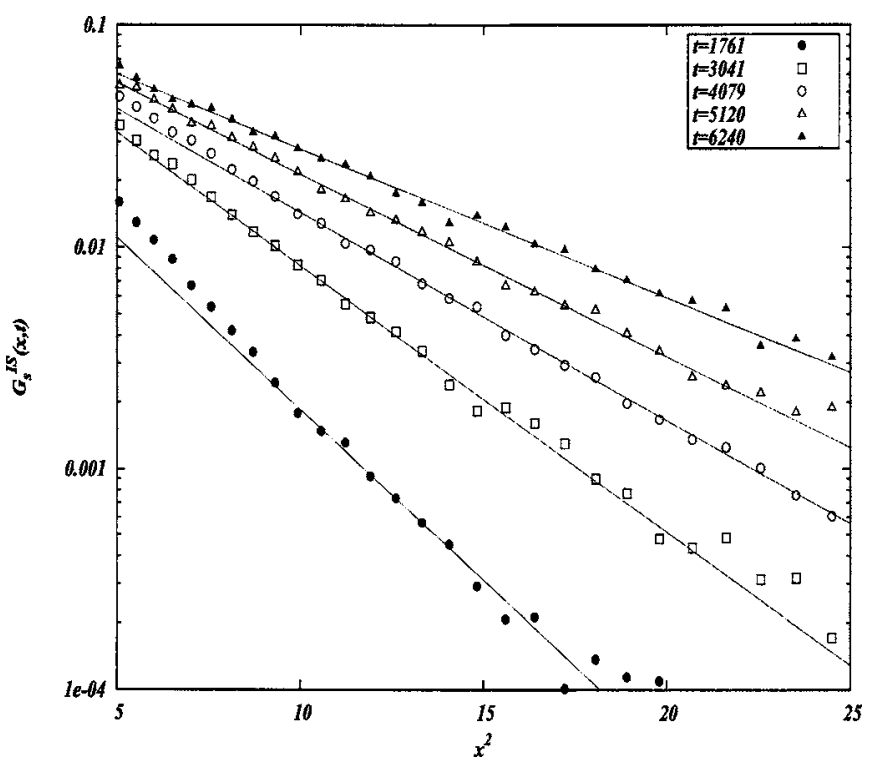

FIG. 4. The van Hove distribution in the Fickian regime. Continuous lines are Gaussian fits.

tween the Fickian and exponential regimes will be analyzed in greater detail in Sec. III.

Recently a frozen regime was also observed in spin models of glasses with kinetic constraints. ${ }^{15}$ In this regime a fraction of the system remains frozen up to times which increase rapidly with decreasing temperature, as described by a persistence function. In the van Hove distribution the frozen component is reflected by a delta peak at the origin. In molecular systems a strictly frozen component cannot be seen due to vibrations. But from the inherent structures dynamics we obtained a delta peak for our smallest sample of $N$ $=130$ particles. In this case we see that the whole system remains frozen for some samples up to times of the order of the typical relaxation time. But once a particle moves, all the others also move, although the displacements can be very small. As the size of the system grows, completely frozen samples became rare. For the 10000 particle system we have not seen the occurrence of a frozen sample or of a frozen region within the samples, i.e., it is not possible to observe strictly frozen groups of particles. As the system size grows, the minimum possible displacement of the particles shifts continuously toward smaller distances. At most we must expect to see groups of particles with distinctive mobility, i.e., slower and faster particles.

\section{THE SELF SCATTERING FUNCTION AND CHARACTERISTIC TIME-LENGTH SCALES OF HETEROGENEITIES}

Assuming isotropy of space we computed the following one-dimensional self incoherent scattering function:

$$
F_{s}^{\mathrm{IS}}(k, t)=\frac{1}{N} \sum_{i=1}^{N} \exp \left[i k\left(x_{i}^{\mathrm{IS}}(t)-x_{i}^{\mathrm{IS}}(0)\right)\right] .
$$

in which the superscript IS means that the function is calculated from inherent structures configurations.

In Fig. 5 we show four selected curves corresponding to

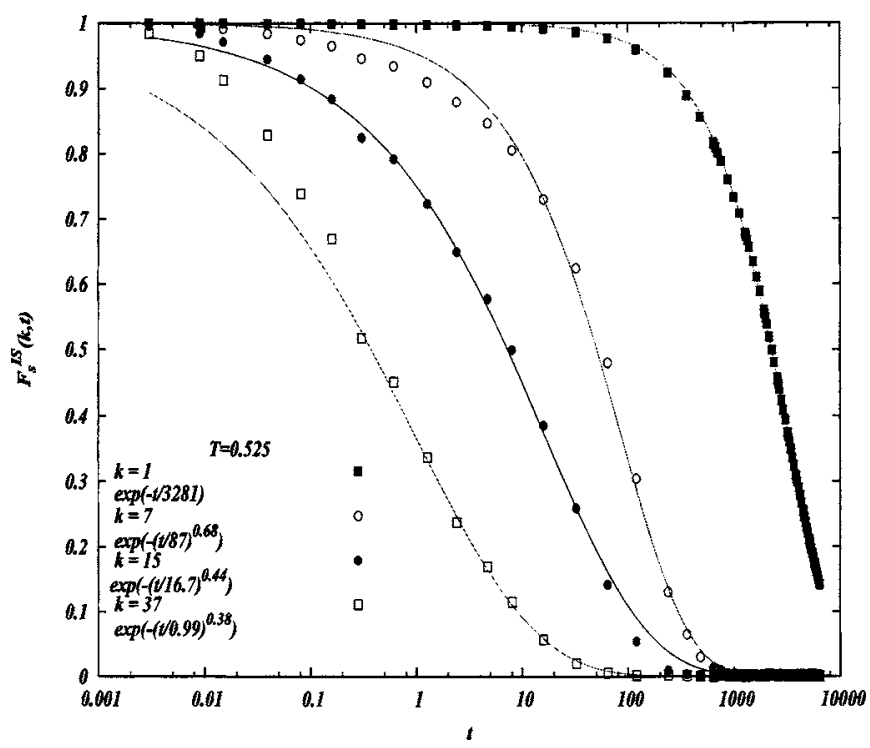

FIG. 5. The self incoherent scattering function for four different wave vectors characteristic of different scaling regimes. In the legend are the corresponding fitting functions (continuous lines).

different wave vectors in order to illustrate the different regimes of relaxation. For small $k$ the relaxation is exponential corresponding to Fickian diffusion of these modes. For wave vectors around the peak of the structure factor $(k=7$ is shown in the figure) or larger, the long time decay can be well fitted by a stretched exponential with a $k$ dependent exponent which will be analyzed in the following. The regime of large $k$ 's correponds to the system being around the basin of a single inherent structure, and the decay can be fitted with two stretched exponentials, one for the short time and another for the long time regimes of the relaxation. This analysis was performed and discussed in detail in Refs. 40 and 41. In Fig. 6 we show the main result of this work, the dependence of the relaxation time $\tau(k)$ on wave vector. Relaxation times for

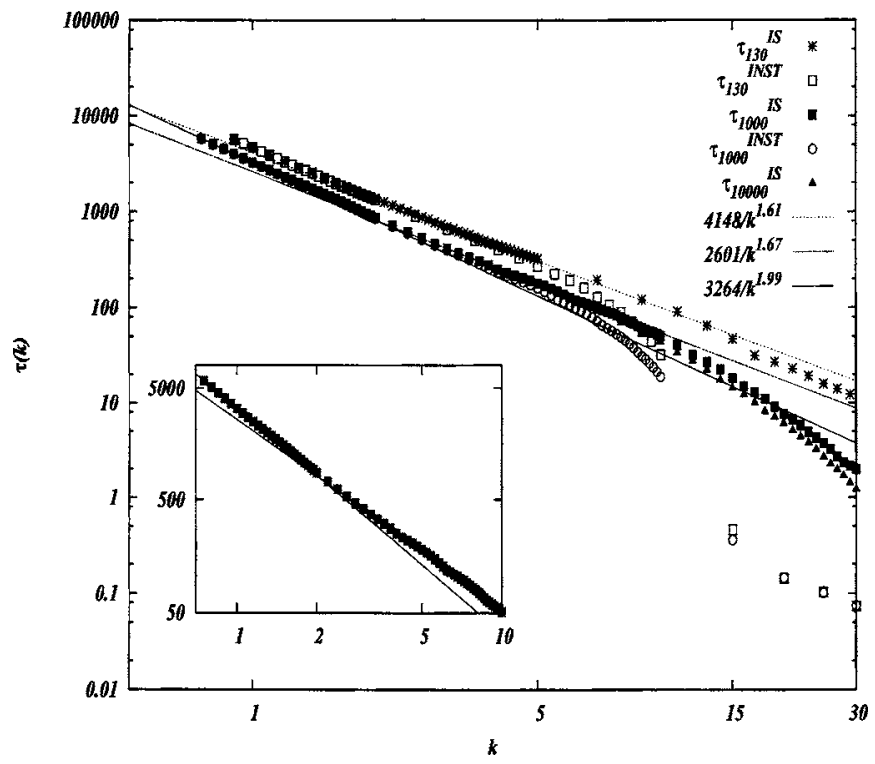

FIG. 6. Wave vector dependence of relaxation times for three different sizes, inherent and instantaneous dynamics. Different scaling regimes are indicated with full lines. Inset: zoom of the Fickian crossover region. 


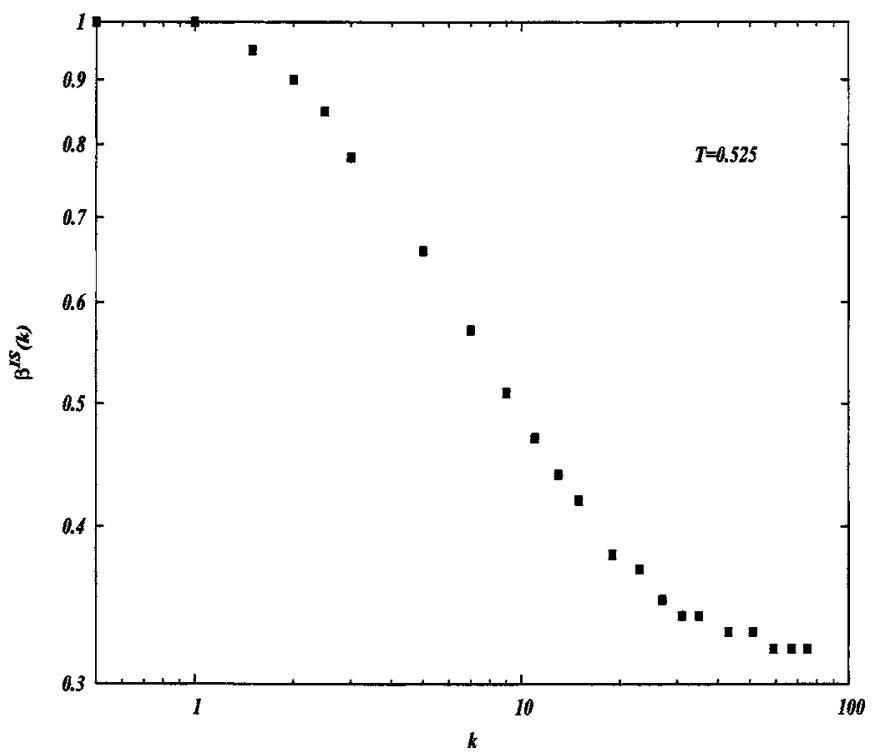

FIG. 7. Wave vector dependence of the stretching exponent $\beta$.

each $k$ were obtained as usual, as the time at which the self scattering function decays to $1 / e$ of its initial value. In this plot we compare the results from inherent structures (IS) and real coordinates for three system sizes: $N=130, N=1000$, and $N=10000$. We see that the data for the two larger systems almost coincide, so one can be confident that already for $N=1000$ there are no finite size effects in the scaling regimes. This figure is very interesting, ${ }^{44}$ showing different scaling regimes between time and length scales. For each system size and for the smallest wave vectors, the results from IS and real coordinates coincide, while they differ strongly at large wave vectors, as expected.

For wave vectors $k \leqslant 2$ and times $\tau \geqslant 1000$, a Gaussian scaling $\tau \simeq 1 / k^{2}$ is observed: this is the Fickian diffusion regime (see the inset in Fig. 6). At $k \approx 2$ a crossover to another scaling form is observed: the system enters an anomalous diffusion regime with scaling $\tau \simeq 1 / k^{1.6}$. This regime extends between $2 \leqslant k \leqslant 10$ and times between $50 \leqslant \tau$ $\leqslant 1000$. This is the regime in which the dynamics is heterogeneous and the relaxation of correlation functions is stretched. Comparing the length and time windows in this regime we realize that they correspond to the exponential decay of the van Hove distribution (see Fig. 8).

For $k \geqslant 10$ the relaxation times decay rapidly in a narrow interval of wave vectors. Note that this effect of rapid decay is much more pronounced in the data corresponding to the real coordinates than in the inherent structures data. This is due to the cage effect, in which the diffusion is temporarily halted, seen in the figure as a narrow interval in $k$ values. For scales larger than $k \geqslant 20$ (data not shown), a linear scaling works well: the diffusion is ballistic. While the ballistic regime is nicely observed in the real dynamics, the corresponding curves for the inherent structures dynamics show instead a slow decay of relaxation times with $k$, because the fast motion of the particles is filtered out. The second crossover, between the subdiffusion and the faster decay, happens at a time of the order of the alpha relaxation time. This time

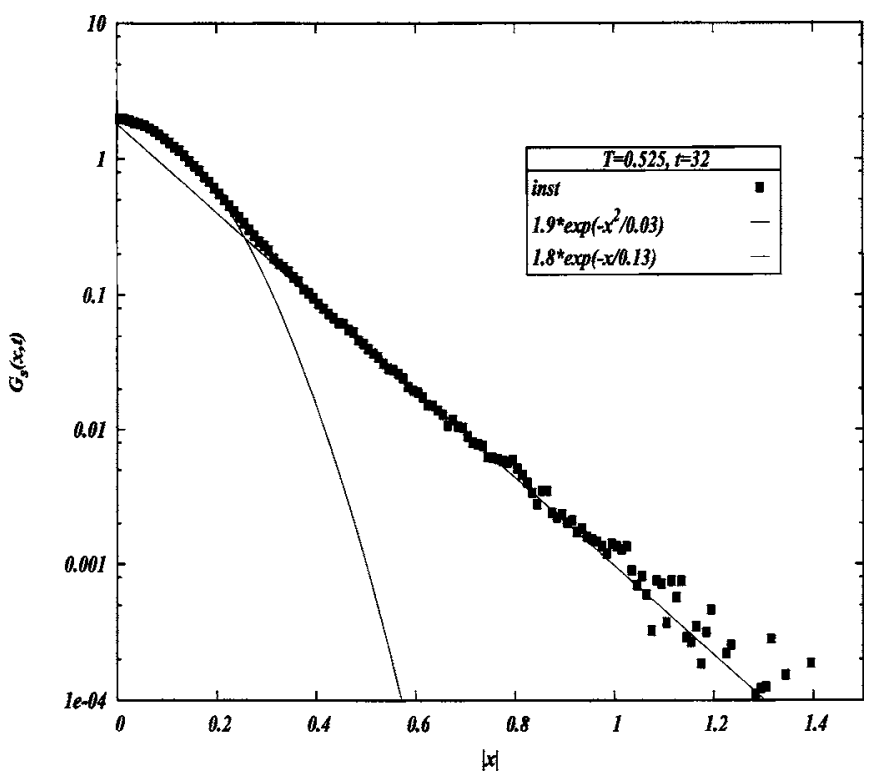

FIG. 8. The van Hove distribution from instantaneous coordinates at $t=32$. The data can be fitted by a Gaussian at small distances plus an exponential decay at larger distances.

corresponds to the peak in the four point susceptibility and the corresponding length scale to the typical size of heterogeneities.

The crossover between Fickian and anomalous diffusion at nearly $k_{F} \simeq 2$ allows one to define a characteristic length scale $l_{F} \simeq 3$ as the scale for the onset of Fickian diffusion at the temperature considered. Below this scale cooperativity rules the motion of individual particles and the dynamics is heterogeneous. This length scale corresponds to the maximum size of heterogeneities in the system. The departure from anomalous diffusion at nearly $k_{H} \simeq 10$ allows one to define a second length scale $l_{H} \simeq 0.6$, as the minimum size of heterogeneous regions. Note that this size is very small at this temperature. It would be extremely interesting to repeat this analysis for different temperatures approaching and crossing the mode coupling transition temperature.

In Fig. 7 we show the dependence of the stretching exponent $\beta^{\mathrm{IS}}(k)$ on wave vector. It decreases monotonously from exponential ( $\beta=1$, Fickian) behavior at small $k$ to an apparent saturation around $\beta \simeq 0.3$ at large $k$. Due to the complex $t$ and $k$ dependence of the stretching exponent, we were not able to collapse the data of the self scattering function for different wave vectors onto a single master curve.

\section{EXPERIMENTAL EVIDENCE}

There is a relatively large literature reporting results on heterogeneous dynamics in glasses and the search for characteristic time and length scales. ${ }^{19,22}$ Nevertheless there are, to our knowledge, only a few papers reporting detailed measurements of particle displacements in supercooled liquids and glasses. ${ }^{20,21,42}$

In Fig. 8 we show that in the real dynamics, for a time less than the alpha relaxation time, the van Hove function can be fitted by a Gaussian contribution from vibrations at small distances plus an exponential contribution at large distances. This figure can be compared, e.g., with Fig. 3 of 
Ref. 21, where experimental data from colloidal supercooled fluids and glasses were fitted with a stretched exponential with exponent $\simeq 0.8$, although an exponential fit can probably work also in that case.

In another, similar experiment, Kegel et al., ${ }^{20}$ using time-resolved fluorescence confocal scanning microscopy, also measured the self part of the van Hove function. They classified the particles in two subsets: one fast and one slow, and fitted the data for both subsets with two Gaussians. The fits are very good, as recently observed for the same time scale in simulations of the LJBM. ${ }^{43}$ The difference in the behavior of the distribution function between both experimental results probably reflects the different time regimes in which the measurements were done in each case. It would be very interesting to have accurate measurements in the whole time span between the beta relaxation scales up to several times the alpha scale in order to get from that an estimation of the characteristic length scales discussed in this work.

\section{CONCLUSION AND PERSPECTIVES}

The mechanisms of particle diffusion in deeply supercooled liquids are still poorly understood. In particular, the region of intermediate time and length scales, in which diffusion is anomalous, is still waiting for a complete theoretical description. In the meantime, new highly precise measurements probing local dynamics and extensive computer simulations are giving important insights into the basic mechanisms that underlie particle motion. By means of molecular dynamics simulation on a Lennard-Jones supercooled liquid we showed that the van Hove distribution and self scattering functions still bring us new and rich information. They show at least three well defined regimes on different time and length scales. On very short times and lengths the motion of particles is ballistic. Then heterogeneities develop and at times of the order of the relaxation time a well defined crossover length $l_{H}$ can be obtained from the $k$ dependence of the relaxation times. This length corresponds to the typical size of spatial heterogeneities. In this region the diffusion is anomalous and time correlations decay in a stretched exponential way. Also in this space-time regime the van Hove distribution shows a well-defined exponential decay. To our knowledge this exponential decay has not been observed or analyzed in other models, like the much studied kinetically constrained lattice models, but is clearly present in experimental results on colloidal systems. At very long time scales, several orders larger than the alpha relaxation scale, Fickian diffusion sets in, and the distribution of displacements slowly converges to a Gaussian. The crossover from heterogeneous to Fickian dynamics is also clearly observed in the $k$ dependence of the self scattering function, and a second typical length $l_{F}$ can be obtained. Scaling forms typical of normal and anomalous diffusion can be seen for distances larger and smaller than $l_{F}$, respectively. An interesting study on the temperature dependence of the onset time for Fickian diffusion was recently done by Szamel et al. ${ }^{31}$ In that work the onset time for Fickian diffusion is defined as the time at which a fixed deviation from Gaussianity was observed in the distribution of the logarithm of particle displacements
$P\left(\log _{10} \delta r, t\right)$. Its temperature dependence was analyzed and compared with the corresponding behavior of other characteristic time scales. A particularly interesting result is the observation that the ratio between the Fickian diffusion onset time $\tau_{F}$ and the alpha relaxation time $\tau_{\alpha}$ grows with decreasing temperature, but tends to saturate near the mode coupling transition temperature $T_{c}$. This may indicate that the mechanisms behind both time scales are essentially the same as the crossover temperature is approached.

Although the identification of characteristic dynamical lengths can be naturally introduced through four point correlation and response functions, we have shown that the relevant information can already be obtained from two point functions, like the van Hove distribution and self scattering correlation functions. To understand the emergence of the different regimes observed in the simulations from a single microscopic model, or unified theoretical framework, is still a big challenge.

\section{ACKNOWLEDGMENTS}

D.A.S. wishes to thank L. Berthier for interesting discussions and comments. This work was partly supported by Centra Latinoamericano de Física (CLAF), CONICET and ANPCyT (Argentina), CNPq and CAPES (Brazil), and ICTP (Italy) through Grant No. Net-61 Latinamerican Network on Slow Dynamics in Complex Systems.

${ }^{1}$ P. Debenedetti, Metastable Liquids, Concepts and Principles (Princeton University Press, Princeton, 1996).

${ }^{2}$ P. G. Debenedetti and F. H. Stillinger, Nature (London) 410, 259 (2001).

${ }^{3}$ A. Cavagna, Europhys. Lett. 53, 490 (2001).

${ }^{4}$ T. S. Grigera, A. Cavagna, I. Giardina, and G. Parisi, Phys. Rev. Lett. 88, 55502 (2002).

${ }^{5}$ S. Büchner and A. Heuer, Phys. Rev. Lett. 84, 2168 (2000)

${ }^{6}$ B. Doliwa and A. Heuer, Phys. Rev. Lett. 91, 235501 (2003).

${ }^{7}$ L. Angelani, G. Ruocco, M. Sampoli, and F. Sciortino, J. Chem. Phys. 119, 2120 (2003).

${ }^{8}$ L. Angelani, R. D. Leonardo, G. Ruocco, A. Scala, and F. Sciortino, Phys. Rev. Lett. 85, 5356 (2000).

${ }^{9}$ D. Wales, Energy Landscapes (Cambridge University Press, Cambridge, 2003).

${ }^{10}$ F. Ritort and P. Sollich, Adv. Phys. 52, 219 (2003).

${ }^{11}$ J. P. Garrahan and D. Chandler, Phys. Rev. Lett. 89, 035704 (2002).

${ }^{12}$ L. Berthier and J. P. Garrahan, Phys. Rev. E 68, 041201 (2003).

${ }^{13}$ Y. Jung, J. P. Garrahan, and D. Chandler, Phys. Rev. E 69, 061205 (2004)

${ }^{14}$ S. Whitelam, L. Berthier, and J. P. Garrahan, Phys. Rev. Lett. 92, 185705 (2004).

${ }^{15}$ L. Berthier, D. Chandler, and J. P. Garrahan, Europhys. Lett. 69, 230 (2005)

${ }^{16}$ W. Götze, Condens. Matter Phys. 1, 873 (1998).

${ }^{17}$ W. Götze and L. Sjögren, Rep. Prog. Phys. 55, 241 (1992).

${ }^{18}$ S. P. Das, Rev. Mod. Phys. 76, 785 (2004).

${ }^{19}$ M. D. Ediger, Annu. Rev. Phys. Chem. 51, 99 (2000)

${ }^{20}$ W. K. Kegel and A. van Blaaderen, Science 287, 290 (2000).

${ }^{21}$ E. R. Weeks, J. C. Crocker, A. C. Levitt, A. Schofield, and D. A. Weitz, Science 287, 627 (2000).

${ }^{22}$ H. Sillescu, J. Non-Cryst. Solids 243, 81 (1999).

${ }^{23}$ C. Donati, S. Franz, G. Parisi, and S. C. Glotzer, J. Non-Cryst. Solids 307, 215 (2002).

${ }^{24}$ S. C. Glotzer, V. N. Novikov, and T. B. Schroder, J. Chem. Phys. 112, 509 (2000).

${ }^{25}$ S. C. Glotzer, J. Non-Cryst. Solids 274, 342 (2000).

${ }^{26}$ C. Toninelli, M. Wyart, L. Berthier, G. Biroli, and J. P. Bouchaud, Phys. Rev. E 71, 041505 (2005).

${ }^{27}$ L. Berthier, Phys. Rev. E 69, 020201(R) (2004).

${ }^{28}$ A. M. Puertas, M. Fuchs, and M. E. Cates, J. Chem. Phys. 121, 2813 
(2004).

${ }^{29}$ D. R. Reichman, E. Rabani, and P. L. Geissler, J. Phys. Chem. B 109 14654 (2005).

${ }^{30}$ E. Flenner and G. Szamel, Phys. Rev. E 72, 011205 (2005)

${ }^{31}$ G. Szamel and E. Flenner, cond-mat/0508108 (2005).

${ }^{32}$ A. C. Pan, J. P. Garrahan, and D. Chandler, cond-mat/0410525 (2004).

${ }^{33}$ J. P. Hansen and I. R. McDonald, Theory of Simple Liquids, 2nd ed. (Academic, New York, 1994).

${ }^{34}$ W. Kob and H. C. Andersen, Phys. Rev. E 51, 4626 (1995).

${ }^{35}$ C. Y. Liao and S. H. Chen, Phys. Rev. E 64, 031202 (2001).

${ }^{36}$ G. Fabricius and D. A. Stariolo, Phys. Rev. E 66, 031501 (2002).

${ }^{37}$ R. A. Denny, D. R. Reichman, and J.-P. Bouchaud, Phys. Rev. Lett. 90, 025503 (2003).
${ }^{38}$ M. Vogel, B. Doliwa, A. Heuer, and S. Glotzer, J. Chem. Phys. 120, 4404 (2004).

${ }^{39}$ T. B. Schroder, S. Sastry, J. C. Dyre, and S. C. Glotzer, J. Chem. Phys. 112, 9834 (2000).

${ }^{40}$ G. Fabricius and D. A. Stariolo, Physica A 331, 90 (2004).

${ }^{41}$ D. A. Stariolo, J. J. Arenzon, and G. Fabricius, Physica A 340, 316 (2004).

${ }^{42}$ L. Berthier, G. Biroli, J.-P. Bouchaud, L. Cipelletti, D. El Masri, D. L'Hôte, F. Ladieu, and M. Pierno, Science 310, 1797 (2005).

${ }^{43}$ M. S. Shell, P. G. Debenedetti, and F. H. Stillinger, preprint cond-mat/ 0506608.

${ }^{44}$ This plot is shown in Ref. 15 for the FA kinetic model. 